\title{
Evaluation of an organ-based tube current modulation tool in pediatric CT examinations
}

\author{
Antonios E. Papadakis ${ }^{1} \cdot$ John Damilakis ${ }^{2}$
}

Received: 22 November 2019 / Revised: 16 March 2020 / Accepted: 10 April 2020 / Published online: 20 May 2020

(C) The Author(s) 2020

\begin{abstract}
Objectives To investigate the effect of an organ-based tube current modulation (OTCM) technique on organ absorbed dose and assess image quality in pediatric $\mathrm{CT}$ examinations.

Methods Four physical anthropomorphic phantoms that represent the average individual as neonate, 1-year-old, 5-year-old, and 10-year-old were used. Standard head and thorax acquisitions were performed with automatic tube current modulation (ATCM) and ATCM+OTCM. Dose calculations were performed by means of Monte Carlo simulations. Radiation dose was measured for superficial and centrally located radiosensitive organs. The angular range of the OTCM exposure window was determined for different tube rotation times $(t)$ by means of a solid-state detector. Image noise was measured as the standard deviation of the Hounsfield unit value in regions of interest drawn at selected anatomical sites.

Results ATCM+OTCM resulted in a reduction of radiation dose to all radiosensitive organs. In head, eye lens dose was reduced by up to $13 \%$ in ATCM+OTCM compared with ATCM. In thorax, the corresponding reduction for breast dose was up to $10 \%$. The angular range of the OTCM exposure window decreased with $t$. For $t=0.4 \mathrm{~s}$, the angular range was limited to $74^{\circ}$ in head and $135^{\circ}$ for thorax. Image noise was significantly increased in ATCM+OTCM acquisitions across most examined phantoms $(p<0.05)$.

Conclusions OTCM reduces radiation dose to exposed radiosensitive organs with the eye lens and breast buds exhibiting the highest dose reduction. The OTCM exposure window is narrowed at short $t$. An increase in noise is inevitable in images located within the OTCM-activated imaged volume.

Key Points

- In pediatric CT, organ-based tube current modulation reduces radiation dose to all major primarily exposed radiosensitive organs.

- Image noise increases within the organ-based tube current modulation enabled imaged volume.

- The angular range of the organ-based tube current modulation low exposure window is reduced with tube rotation time.
\end{abstract}

Keywords Tomography, x-ray computed $\cdot$ Child $\cdot$ Radiation dosage $\cdot$ Monte Carlo method $\cdot$ Radiation protection

\section{Abbreviations}

ASIR Adaptive statistical iterative reconstruction

ATCM Automatic tube current modulation

$\mathrm{CTDI}_{\mathrm{vol}}$ Volume computed tomography dose index

DICOM Digital Imaging and Communications in Medicine

MC Monte Carlo

Antonios E. Papadakis

apapadak@pagni.gr

1 Department of Medical Physics, University Hospital of Heraklion, Stavrakia, 71110 Heraklion, Crete, Greece

2 Department of Medical Physics, University of Crete, Medical School, Stavrakia, 71110 Heraklion, Crete, Greece
OTCM Organ-based tube current modulation

PMMA Polymethyl methacrylate

ROI Region of interest

$t \quad$ Tube rotation time

\section{Introduction}

The lens of the eye and breast are considered among the most radiosensitive tissues of the human body. Based on new epidemiological evidence on the detrimental effects of ionizing radiation, the International Commission on Radiological Protection (ICRP) has recently reduced the radiation dose threshold for cortical and posterior subcapsular cataract 
formation to $500 \mathrm{mGy}$ [1-4]. ICRP has also increased the breast tissue weighting factor for effective dose estimation from 0.05 to 0.12 [2]. These changes suggest that the lens of the eye and breast may be more radiosensitive than previously considered. Increased attention should thus be given to minimize radiation dose to these tissues, especially in children that are considered more radiosensitive than adults and are more likely to undergo multiple $\mathrm{CT}$ examinations during their lifetime [5].

Organ-based tube current modulation (OTCM) techniques reduce the $\mathrm{x}$-ray tube current $(\mathrm{mA})$ over the anterior part of the patient's body circumference aiming to minimize radiation exposure to superficial radiosensitive organs such as eyes, thyroid, and breasts. Different CT vendors have adopted different approaches to implement OTCM. In one approach, implemented by Siemens Healthcare with X-CARE, $\mathrm{mA}$ is reduced when the $\mathrm{x}$-ray tube rotates over the anterior quadrant of the body circumference, while it is increased over the lateral and posterior quadrants to preserve image quality [6-9]. However, an increase in dose absorbed by posterior located radiosensitive organs has been demonstrated [6-8]. In a second approach, implemented by GE Medical Systems with ODM, $\mathrm{mA}$ is reduced when the $\mathrm{x}$-ray tube rotates over the anterior part of the patient's body without increase over the remaining lateral and posterior parts. However, this approach has been documented to deliver images of increased noise [10, 11]. Previous studies performed in adult patients have shown that OTCM may substantially reduce radiation dose to superficial radiosensitive organs $[6,8,12,13]$. To our knowledge, there is scarce published data on the effect of OTCM on radiation dose to superficial radiosensitive organs and image quality in pediatric CT examinations $[14,15]$.

The purpose of this study was to investigate the effect of an OTCM technique on radiation dose to major radiosensitive organs and assess image quality in pediatric $\mathrm{CT}$ examinations.

\section{Materials and methods}

\section{Anthropomorphic phantoms}

Four physical anthropomorphic phantoms (ATOM Phantoms, CIRS) that simulate the average pediatric individual as neonate, 1-year-old, 5-year-old, and 10-year-old were used [16, 17].

\section{Organ-based tube current modulation technique}

Acquisitions were performed on a 64-detector row CT scanner (Revolution GSI, GE Medical Systems). This scanner is equipped with OTCM (ODM, GE Medical Systems). OTCM constitutes a tube current modulation mode that reduces the $\mathrm{mA}$ when the tube travels across the anterior arch of the patient's circumference without increasing it over the remaining lateral and posterior arches. To enable OTCM, the automatic tube current modulation (ATCM) system (AutomA and SmartmA, GE Medical Systems) needs also to be activated. In head, the $\mathrm{mA}$ is reduced by up to $30 \%$ across $90^{\circ}$ anterior projections, while in body, the $\mathrm{mA}$ is reduced by up to $40 \%$ across $180^{\circ}$ anterior projections (Fig. 1) [18].

\section{CT examination protocols}

Head, thorax, and whole body acquisitions were performed using the scanning parameters listed in Table 1 [19]. Each anatomical region was first scanned with ATCM and then with ATCM+OTCM. All scans were repeated five times to calculate arithmetic averages of the modulated $\mathrm{mA}$ values among scans.

\section{Monte Carlo simulation}

Three-dimensional radiation dose distributions were generated using a MC simulation tool (ImpactMC, CT Imaging $\mathrm{GmbH}$ ) [20-23]. Whole body, $2.5 \mathrm{~mm}$ thick, CT image series $(512 \times 512$ pixels/image $)$ of the physical phantoms were used as input to create whole body age-specific voxelized phantoms. The employment of whole body voxel phantoms is essential to take into account the contribution of scattered radiation from anatomical regions beyond the imaged volume [20]. Simulations were performed by applying density and material segmentation, and using the scanner geometry, $\mathrm{x}$ ray beam energy spectrum, beam filtration, and geometrical characteristics of small and large bowtie filters for head and thorax, respectively (Fig. 2). Data regarding the geometric characteristics of the bowtie filters were obtained from the manufacturer. Thirty-six tube focus positions were simulated per tube rotation. Acquisitions were simulated from vertex to top of $\mathrm{C} 1$ lamina for head, and lung apices to 12th rib for thorax. Simulations were first performed with ATCM and then with ATCM+OTCM. In ATCM, the $\mathrm{mA}(\mathrm{z})$ profile derived from the mean $\mathrm{mA}$ values listed in the images' DICOM header was used as input. In ATCM+OTCM, the mA was reduced for the anterior angles that spanned the eyes for head and breast for thorax. For head, $\mathrm{mA}$ was reduced by $30 \%$ across 9 out of $36\left(90^{\circ}\right)$ simulated tube positions. For thorax, $\mathrm{mA}$ was reduced by $40 \%$ across 18 out of $36\left(180^{\circ}\right)$ simulated tube positions. The $\mathrm{mA}$ values across the remaining posterior projections were those prescribed by the DICOM header. Following MC simulation, an output color-coded dose image matrix series $(512 \times 512$ pixels/image $)$ was generated. These matrices depict the normalized to free-in-air $\mathrm{CTDI}_{\text {air }}(\mathrm{mGy} /$ $\mathrm{mGy} \cdot 100 \mathrm{mAs})$ dose distribution $\left(\mathrm{ND}_{\text {air }}\right)$ imparted in the phantom's body, in voxel-to-voxel correspondence to input $\mathrm{CT}$ images. Measured dose values were normalized $\left(\mathrm{ND}_{\mathrm{m}}\right)$ to $\mathrm{CTDI}_{\mathrm{vol}}(\mathrm{mGy} / 100 \mathrm{mAs})$ for $16 \mathrm{~cm}$ diameter phantom; $\mathrm{ND}_{\mathrm{m}}=\left(\mathrm{ND}_{\mathrm{air}} / \mathrm{CTDI}_{\mathrm{vol}}\right)$. 
Fig. 1 A graphical illustration of the configuration setup used in the MC simulation experiments showing the positioning of the phantom with regard to the angle of OTCM $\mathrm{mA}$ reduction. For head, the $\mathrm{mA}$ was reduced by $30 \%$ in 9 out of 36 tube positions, while for body, the $\mathrm{mA}$ was reduced by $40 \%$ in 18 out of 36 tube positions

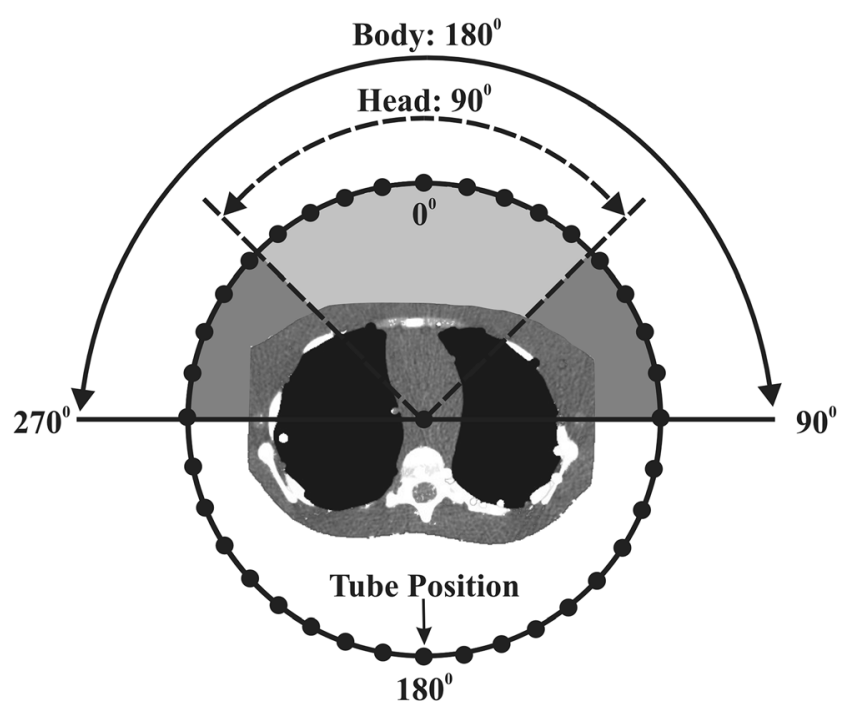

in the anterior chest wall of left and right breast. This ROI size was kept constant for neonate, 1-year-old and 5-yearold phantoms, since breast bud size is considered to be the same until the onset of puberty. Moreover, this size is considered to be identical in boys and girls. To take into account breast development at the onset of girl's puberty, ROI size was increased to $80 \mathrm{~mm}^{2}$ for 10 -year-old phantom. ROIs for eye lens and breast buds were drawn in single images considering that these organs are not expected to extend more than $2.5 \mathrm{~mm}$ along $\mathrm{z}$-axis. The size and position of all other organs was determined in accordance with previously published data [24]. In head, all examined organs were fully encompassed in the simulated scanned volume. In thorax, breast buds and lungs were fully encompassed; thyroid and kidneys were outside, while
Table 1 Examination protocols with acquisition and reconstruction parameters for head and thorax pediatric routine CT examinations. All acquisitions were performed in the helical mode. Head acquisitions were performed in the caudocranial direction from the base of the skull to the superior limit of the skull at a pitch of 0.531 . Thorax acquisitions were performed in the craniocaudal direction from the apex of the lungs to the end of the diaphragm at a pitch of 1.375 . Images were reconstructed using the "standard" reconstruction filter kernel, the adaptive statistical iterative reconstruction (ASIR) at the $40 \%$ level, and a slice thickness of $2.5 \mathrm{~mm}$

\begin{tabular}{|c|c|c|c|c|c|c|c|}
\hline & Scout view & Scan field of view & $\mathrm{kVp}$ & Rot. time (sec) & Beam coll. (mm) & $\min \mathrm{mA} / \mathrm{max} \mathrm{mA}$ & Noise index \\
\hline \multicolumn{8}{|l|}{ Head } \\
\hline Neonate & $\mathrm{AP}+\mathrm{LAT}$ & Pediatric head & 80 & 0.5 & 20 & $100 / 350$ & 5.80 \\
\hline 1 year & $\mathrm{AP}+\mathrm{LAT}$ & Pediatric head & 100 & 0.5 & 20 & $100 / 350$ & 7.70 \\
\hline 5 years & $\mathrm{AP}+\mathrm{LAT}$ & Small head & 120 & 0.5 & 20 & $100 / 350$ & 5.14 \\
\hline 10 years & $\mathrm{AP}+\mathrm{LAT}$ & Small head & 120 & 1.0 & 20 & $100 / 350$ & 3.86 \\
\hline \multicolumn{8}{|l|}{ Thorax } \\
\hline Neonate & $\mathrm{AP}+\mathrm{LAT}$ & Pediatric body & 80 & 0.4 & 20 & $25 / 300$ & 11.6 \\
\hline 1 year & $\mathrm{AP}+\mathrm{LAT}$ & Small body & 80 & 0.4 & 20 & $25 / 300$ & 11.6 \\
\hline 5 years & $\mathrm{AP}+\mathrm{LAT}$ & Small body & 80 & 0.4 & 20 & $25 / 300$ & 10.7 \\
\hline 10 years & AP+LAT & Large body & 80 & 0.4 & 40 & $25 / 300$ & 13.11 \\
\hline
\end{tabular}

Whole body acquisition was performed using the exposure parameters prescribed for thorax acquisition for each anthropomorphic phantom 


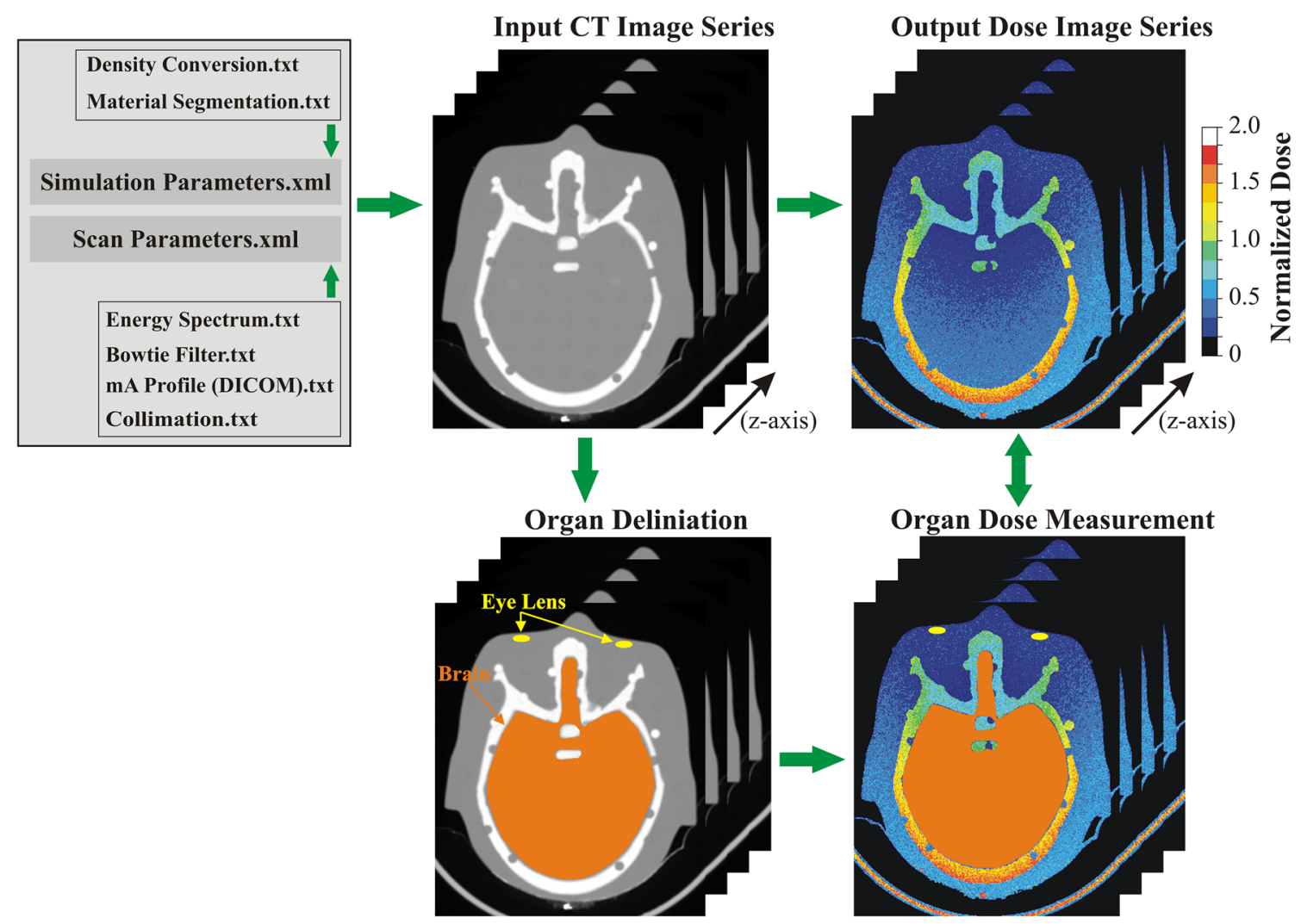

Fig. 2 A schematic diagram of the MC simulation algorithm employed. Required data on simulation density conversion and material segmentation along with data on scan parameters including energy spectrum, bowtie filter, $\mathrm{mA}(\mathrm{z})$ profile derived from DICOM header,

liver was partially encompassed in the simulated scanned volume. ROIs per input CT image slice (ROI(i)) were transferred to the corresponding output dose matrices. The mean dose image pixel (MDP(i)) and its standard deviation (SD) from each ROI were recorded. MDP(i) was the dose absorbed for the fraction of the organ depicted in that particular image. The total organ dose (TOD) was estimated as the sum of the area-weighted average of MDP(i) values obtained from all images where the organs were depicted;

$\mathrm{TOD}=\left(\frac{\sum \mathrm{MDP}(i) \times \mathrm{ROI}(i)}{\sum \mathrm{ROI}(i)}\right)$

To take into account the error in organ dose measurement related to the location of each radiosensitive organ, each ROI was drawn ten times at slightly different locations along the $x-y$ plane of the axial image. The error in estimated organ dose was calculated through error propagation using the standard deviation of the average measured organ doses and the recorded SDs of the MIP(i) values. For comparison of estimated doses, the Student's $t$ test for paired samples was used. A significant difference was set at $p<0.05$. and collimation are input in the form of txt files. Additional parameters such as irradiation geometry, maximum number of simulated photons and energy level below which simulated photons are considered absorbed are also predefined

\section{OTCM: the effect of tube rotation time $(t)$ on tube output}

To investigate the potential effect of $t$ on OTCM scheme, the tube output was measured using an $\mathrm{x}$-ray multimeter equipped with a solid-state point detector (Black Piranha, CT Dose Profiler, RTI Electronics). This detector is capable of acquiring up to 2000 exposure measurements/s. ATCM+OTCM acquisitions were performed at $0.4,0.5$, $0.7,1$, and $2 \mathrm{~s}$. The standard head, $16 \mathrm{~cm}$ diameter, polymethyl methacrylate (PMMA) phantom (CTPhantom, IBA Dosimetry) was scanned using the examination protocols applied for pediatric head and thorax under fixed mA, ATCM, and ATCM+OTCM (Table 2). This phantom was selected because it is cylindrical, provides uniform attenuation across $360^{\circ}$ and is used as reference for $\mathrm{CTDI}_{\mathrm{vol}}$ reporting in pediatric patients. In fixed $\mathrm{mA}$ acquisition, the $\mathrm{mA}$ was manually adjusted to reach a $\mathrm{CTDI}_{\mathrm{vol}}$ that matches the corresponding $\mathrm{CTDI}_{\mathrm{vol}}$ of ATCM-activated acquisition. AP and LAT scout views of the phantom were acquired. The phantom was then removed from the table and the solid-state detector was positioned, free-in-air, at the gantry isocenter. A floormounted arm was used to keep the detector stationary 
throughout the scan. Exposure rate profiles as a function of tube projection angle were recorded. This configuration facilitates free-in-air measurement of the tube output based on the scout view predetermined $\mathrm{mA}$-modulated profiles.

\section{Quantitative image quality assessment}

To assess the effect of OTCM on image quality, we have compared the noise in images obtained from ATCM and ATCM+ OTCM-activated acquisitions. Image noise was measured as the SD of the mean Hounsfield unit (HU) value in $\sim 3 \mathrm{~cm}^{2}$ ROIs. These ROIs were drawn at various locations over uniform brain and soft tissue equivalent areas (Fig. 3). Ten ROIs were drawn in the images obtained with ATCM at the level depicting eyes for head and middle heart for thorax. These ROIs were pasted to the corresponding images obtained with ATCM+OTCM. To reduce measurement error, each parameter was measured five times on five consecutive images. Quantitative image analysis was performed using ImageJ $(1.48 \mathrm{v}$, NIH). For noise comparison, the Student's $t$ test was used for paired samples. A significant difference was set at $p<0.05$.

\section{Results}

In ATCM-activated head acquisitions, the absorbed dose to the eye lens ranged from $3.6 \mathrm{mGy}$ for neonate to $27.6 \mathrm{mGy}$ for 10 -year-old phantom (Table 3). The corresponding absorbed doses in ATCM+OTCM-enabled acquisitions ranged from 3.1 to $25.0 \mathrm{mGy}$, resulting in $13 \%$ and $9 \%$ reduction, respectively. In ATCM-activated thorax acquisitions, the absorbed dose to the breast buds ranged from $0.55 \mathrm{mGy}$ for neonate to $1.3 \mathrm{mGy}$ for 5 -year-old phantom. The corresponding absorbed doses in ATCM+OTCM-enabled acquisitions ranged from 0.53 to $0.95 \mathrm{mGy}$, resulting in $4 \%$ and $27 \%$ reduction, respectively. Organ dose was slightly increased when the angular range of the OTCM scheme was reduced, with this increase, however, being not statistically significant (Table 4).

Measurements of exposure rate on the $16 \mathrm{~cm}$ diameter PMMA phantom confirmed that OTCM reduces exposure (at $0^{\circ}$ ) by $30 \%$ in head and $40 \%$ in thorax. In head, the angular range of OTCM window increased from $74^{\circ}$ for $0.4 \mathrm{~s}$ to $95^{\circ}$ for $2 \mathrm{~s}$. In thorax, the corresponding increase was from $135^{\circ}$ to $170^{\circ}$. Shown in Fig. 4 for thorax is the measured exposure rate as a function of projection angle in fixed $\mathrm{mA}$, ATCM, and $\mathrm{ATCM}+\mathrm{OTCM}$ acquisitions (a) and the ATCM+OTCM-activated exposure rate profiles at different $t$ (b).

Image noise was increased in ATCM+OTCM-enabled acquisitions (Table 5). Mean image noise increase was up to $11 \%$ for head and 19\% for thorax (Fig. 5). The increase in image noise was statistically significant $(p<0.05)$ in all but neonate phantom.

\section{Discussion}

To our knowledge, this is the first study aimed to assess the effect of OTCM on radiation dose of radiosensitive organs in children. The presented results showed that OTCM reduces radiation dose to all examined radiosensitive organs and that the angular range of the OTCM low exposure window varies on $t$. While nominal angular ranges prescribed by the manufacturer are $90^{\circ}$ for head and $180^{\circ}$ for thorax, the results of the current study showed that for $t=0.4 \mathrm{~s}$, the low exposure window may be limited to $74^{\circ}$ for head and $135^{\circ}$ for thorax. This suggests that some organs might benefit less from the reduced angular range of the OTCM window. Our results showed however that, although organ dose was slightly increased, this increase was not statistically significant.

Several studies have demonstrated the effect of OTCM on organ dose in CT of adults. In most studies, investigators have employed an OTCM technique that reduces the $\mathrm{mA}$ by $20 \%$ for the anterior $120^{\circ}$ projection views, while increases the $\mathrm{mA}$ for

Table 2 Examination protocol parameters used to measure tube output free-in-air under three operation modes with fixed mA, ATCM, and ATCM+ OTCM exposure of the standard, $16 \mathrm{~cm}$ diameter, head PMMA phantom

\begin{tabular}{|c|c|c|c|c|c|c|c|}
\hline & Scout view & $\begin{array}{l}\text { Scan field } \\
\text { of view }\end{array}$ & $\begin{array}{l}\text { Beam } \\
\text { coll. }(\mathrm{mm})\end{array}$ & $\begin{array}{l}\text { Rot. time } \\
(\mathrm{sec})\end{array}$ & $\mathrm{kVp}$ & Operation mode & \\
\hline \multicolumn{8}{|l|}{ Head } \\
\hline & $\mathrm{AP}+\mathrm{LAT}$ & Pediatric head & 20 & 0.4 & 120 & Fixed $\mathrm{mA}$ & $180 \mathrm{~mA}$ \\
\hline & & & & & & ATCM & NI: 4.5 \\
\hline & & & & & & ATCM+OTCM & NI: 4.5 \\
\hline \multicolumn{8}{|l|}{ Thorax } \\
\hline & $\mathrm{AP}+\mathrm{LAT}$ & Pediatric body & 20 & 0.4 & 80 & Fixed $\mathrm{mA}$ & $35 \mathrm{~mA}$ \\
\hline & & & & & & ATCM & NI: 14 \\
\hline & & & & & & ATCM+OTCM & NI: 14 \\
\hline
\end{tabular}



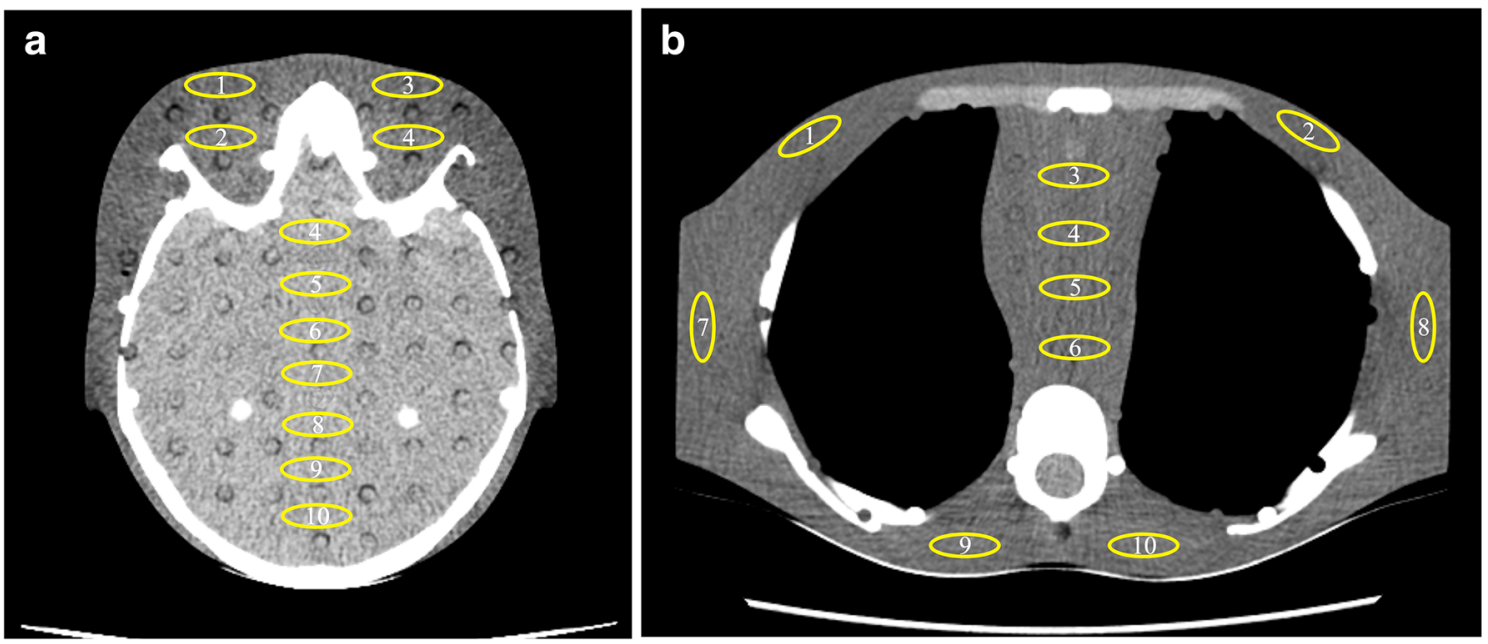

Fig. 3 ROI placement for image noise measurement in ATCM and ATCM+OTCM-activated head (a) and thorax (b) acquisitions. Head and thorax images shown are from the 10-year-old phantom. Window width 100 and window level 30 for (a); window width 400 and window level for 40 (b)

Table $3 \mathrm{CTDI}_{\mathrm{vol}}$ and calculated organ dose values (mGy) in ATCM and ATCM+OTCM-activated head and thorax CT acquisitions. Numbers in parenthesis are percent organ dose reduction

$\mathrm{CTDI}_{\mathrm{vol}}(\mathrm{mGy})$ and organ dose $(\mathrm{mGy}), \mathrm{ATCM}^{*} / \mathrm{ATCM}+\mathrm{OTCM}$

\begin{tabular}{|c|c|c|c|c|}
\hline & Neonate & 1-year-old & 5-year-old & 10-year-old \\
\hline \multicolumn{5}{|l|}{ Head } \\
\hline $\mathrm{CTDI}_{\mathrm{vol}}$ & $11.34 / 10.86$ & $16.67 / 16.25$ & $24.62 / 24.04$ & $49.53 / 47.83$ \\
\hline$\%$ Diff & $-4 \%$ & $-2 \%$ & $-2 \%$ & $-3 \%$ \\
\hline Eye lens & $3.6 \pm 0.06 / 3.1 \pm 0.05$ & $9.0 \pm 0.16 / 7.7 \pm 0.14$ & $18.7 \pm 0.3 / 17.0 \pm 0.3$ & $27.6 \pm 0.5 / 25.0 \pm 0.4$ \\
\hline$\%$ Diff $/ p$ value & $-14 \% /<0.001$ & $-14 \% /<0.001$ & $-9 \% /<0.001$ & $-9 \% /<0.001$ \\
\hline Brain & $3.5 \pm 0.17 / 3.3 \pm 0.05$ & $9.3 \pm 0.46 / 9.0 \pm 0.45$ & $22.6 \pm 1.1 / 22.0 \pm 1.1$ & $41.2 \pm 2.0 / 38.9 \pm 1.9$ \\
\hline$\%$ Diff $/ p$ value & $-6 \% /<0.05$ & $-3 \% / 0.157$ & $-3 \% / 0.238$ & $-5 \% /<0.05$ \\
\hline Salivary glands & $3.7 \pm 0.07 / 3.4 \pm 0.07$ & $10.5 \pm 0.21 / 9.4 \pm 0.19$ & $24.6 \pm 0.5 / 22.2 \pm 0.4$ & $36.3 \pm 0.7 / 33.6 \pm 0.7$ \\
\hline$\%$ Diff $/ p$ value & $-8 \% /<0.001$ & $-10 \% /<0.001$ & $-10 \% /<0.001$ & $-7 \% /<0.001$ \\
\hline \multicolumn{5}{|l|}{ Thorax } \\
\hline $\mathrm{CTDI}_{\mathrm{vol}}$ & $1.87 / 1.70$ & $1.46 / 1.33$ & $2.06 / 1.94$ & $2.82 / 2.73$ \\
\hline$\%$ Diff & $9 \%$ & $9 \%$ & $6 \%$ & $3 \%$ \\
\hline Breast buds & $0.55 \pm 0.009 / 0.53 \pm 0.009$ & $1.1 \pm 0.02 / 0.79 \pm 0.01$ & $1.3 \pm 0.02 / 0.95 \pm 0.016$ & $1.1 \pm 0.02 / 1.0 \pm 0.02$ \\
\hline$\%$ Diff $/ p$ value & $-4 \% / 0.097$ & $-28 \% /<0.001$ & $-27 \% /<0.001$ & $-9 \% /<0.001$ \\
\hline Lung & $0.53 \pm 0.039 / 0.47 \pm 0.035$ & $0.95 \pm 0.070 / 0.85 \pm 0.063$ & $1.5 \pm 0.11 / 1.4 \pm 0.10$ & $1.3 \pm 0.09 / 1.2 \pm 0.09$ \\
\hline$\%$ Diff $/ p$ value & $-11 \% /<0.05$ & $-10 \% /<0.05$ & $-7 \% /<0.05$ & $-8 \% /<0.05$ \\
\hline Liver & $0.43 \pm 0.048 / 0.39 \pm 0.043$ & $0.85 \pm 0.094 / 0.79 \pm 0.088$ & $0.63 \pm 0.069 / 0.61 \pm 0.065$ & $1.1 \pm 0.12 / 1.0 \pm 0.11$ \\
\hline$\%$ Diff $/ p$ value & $-9 \% / 0.065$ & $-7 \% / 0.157$ & $-3 \% / 0.513$ & $-9 \% / 0.067$ \\
\hline Thyroid & $0.25 \pm 0.014 / 0.22 \pm 0.01$ & $0.46 \pm 0.026 / 0.44 \pm 0.025$ & $0.27 \pm 0.015 / 0.25 \pm 0.014$ & $0.28 \pm 0.016 / 0.26 \pm 0.015$ \\
\hline$\%$ Diff $/ p$ value & $-12 \% /<0.001$ & $-4 \% / 0.096$ & $-7 \% /<0.05$ & $-7 \% /<0.05$ \\
\hline Kidneys & $0.25 \pm 0.028 / 0.23 \pm 0.026$ & $0.15 \pm 0.017 / 0.14 \pm 0.016$ & $0.16 \pm 0.018 / 0.16 \pm 0.017$ & $0.68 \pm 0.076 / 0.61 \pm 0.069$ \\
\hline$\%$ Diff $/ p$ value & $-8 \% / 0.115$ & $-6 \% / 0.192$ & - & $-10 \% /<0.05$ \\
\hline
\end{tabular}

*As the mean $\mathrm{mA}$ value listed in the images' DICOM header per z-axis location is averaged over the transversal plane, the resulting $\mathrm{mA}(\mathrm{z})$ profile will represent rather the longitudinal (auto $\mathrm{mA}$ ) than the transversal (smart $\mathrm{mA}$ ) $\mathrm{mA}$ profile. Moreover, the anterior part of the transversal $\mathrm{mA}$ profile will be overestimated through the mean $\mathrm{mA}(\mathrm{z})$ profile, and the lateral part of the transversal $\mathrm{mA}$ profile will be underestimated through the mean $\mathrm{mA}(\mathrm{z})$ profile. Due to the anthropometry of children, this effect increases with the child's age. However, the relative organ dose values listed herein will remain largely unaffected 
Table 4 Calculated organ dose values (mGy) in ATCM+OTCMactivated head and thorax $\mathrm{CT}$ acquisitions of the 5-year-old anthropomorphic phantom at different OTCM schemes. Similar results were found for all anthropomorphic phantoms

\begin{tabular}{lcccc}
\hline Organ dose (mGy), ATCM+OTCM & & & \\
& $90^{\circ}$ & $80^{\circ}$ & $70^{\circ}$ & \\
\hline Head & & & \\
$\quad$ Eye lens & $17.0 \pm 0.3$ & $17.0 \pm 0.3$ & $17.2 \pm 0.3$ & - \\
Brain & $22.0 \pm 1.1$ & $22.2 \pm 1.0$ & $22.2 \pm 1.1$ & - \\
Salivary glands & $22.2 \pm 0.4$ & $22.2 \pm 0.4$ & $22.3 \pm 0.3$ & - \\
& $180^{\circ}$ & $160^{\circ}$ & $140^{\circ}$ & $120^{\circ}$ \\
Thorax & & & & \\
Breast buds & $0.95 \pm 0.016$ & $0.96 \pm 0.014$ & $0.96 \pm 0.012$ & $0.96 \pm 0.016$ \\
Lung & $1.4 \pm 0.10$ & $1.4 \pm 0.11$ & $1.5 \pm 0.12$ & $1.6 \pm 0.11$ \\
Liver & $0.61 \pm 0.065$ & $0.61 \pm 0.075$ & $0.63 \pm 0.071$ & $0.64 \pm 0.070$ \\
Thyroid & $0.25 \pm 0.014$ & $0.26 \pm 0.014$ & $0.25 \pm 0.014$ & $0.26 \pm 0.014$ \\
Kidneys & $0.16 \pm 0.017$ & $0.16 \pm 0.019$ & $0.17 \pm 0.016$ & $0.16 \pm 0.020$ \\
\hline
\end{tabular}

the remaining projections, so that the total exposure during one tube rotation is equal to the exposure without OTCM [25]. This increase is required to preserve image quality. Although this approach has been documented to reduce dose to superficial radiosensitive organs, it provokes a dose increase to centrally and posteriorly located organs. Franck et al [6] have shown that OTCM in thorax CT of females reduces the dose to thyroid and breast by $18 \%$ and $19 \%$, respectively. However, the doses for lung, liver, and kidney were found $17 \%, 11 \%$, and $26 \%$ higher. Ketelsen et al have shown that OTCM reduces breast dose by $35 \%$ without a change in signal-to-noise ratio [26]. Wang et al, in a study using a non-anthropomorphic phantom, have shown that OTCM reduces breast dose by $12 \%$ at an increased, however,
$\mathrm{CTDI}_{\mathrm{vol}}$ [27]. Yamauchi-Kawaura et al have reported that OTCM reduces eye lens and breast dose by $26 \%$ and $17 \%$, respectively, in a 6-year-old anthropomorphic phantom, but increases posterior skin dose by $20 \%$ [15].

Herein, an OTCM technique that reduces the $\mathrm{mA}$ for the anterior without increasing it in the remaining posterior views has been employed. That technique was shown to reduce radiation dose not only to superficial but also to more centrally located organs. The effect of OTCM on radiation dose and image quality using this technique has been presented in two studies. These studies, however, have been limited to adults. Gandhi et al have shown that dose is reduced by $20 \%$ in eye lens and $8 \%$ in brain for head, and 34\% in breast, $20 \%$ in lung,

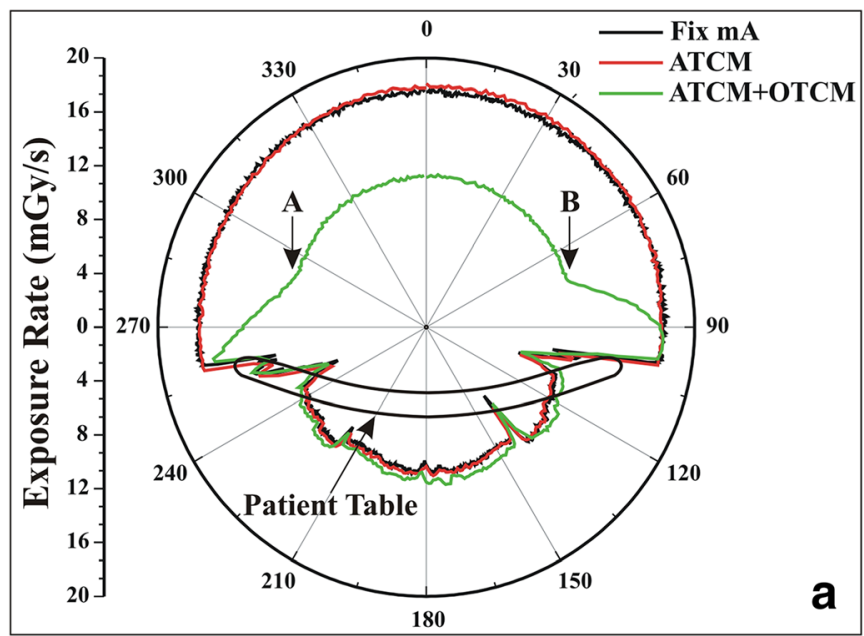

Fig. 4 a Measured exposure rate as a function of tube projection angle in fixed $\mathrm{mA}$, ATCM, and ATCM+OTCM acquisition of the $16 \mathrm{~cm}$ diameter, PMMA phantom using the thorax examination protocol. Tube rotation time was set at $0.4 \mathrm{~s}$. The low exposure rate is applied across a $140^{\circ}$ arch (from point (A) to (B)), while on either side of the low exposure window, there is a $30^{\circ}$ transition range. Exposure rate does not differ between fixed $\mathrm{mA}$ and ATCM due to the circular cross section of the

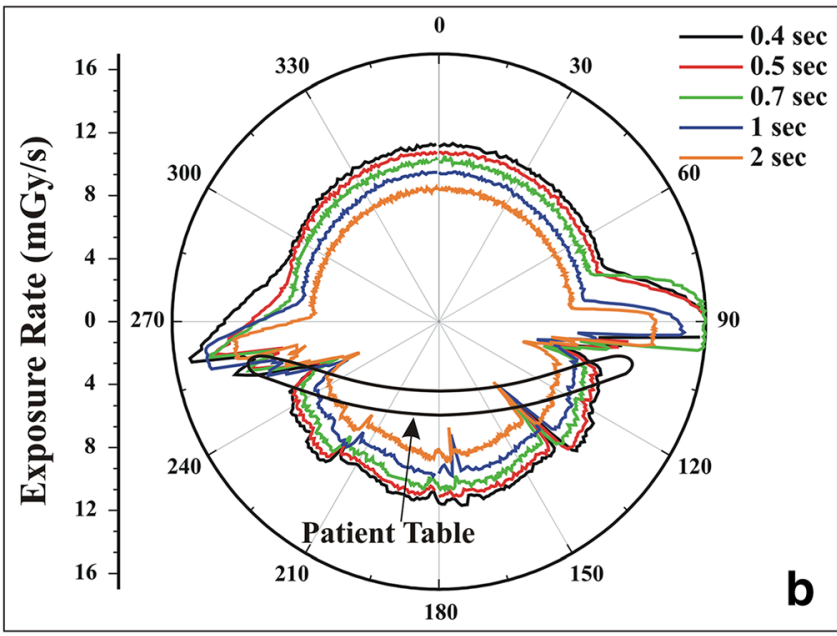

PMMA phantom and the exposure geometry. The abrupt exposure rate changes across the $90^{\circ}$ to $270^{\circ}$ arch originate from changes in attenuation of the patient's table top across tube projections. b Measured exposure rate as a function of tube projection angle in ATCM+OTCM acquisition of the $16 \mathrm{~cm}$ diameter, PMMA phantom at different $(t)$ for thorax examination protocol 
Table 5 Image noise values measured in ROIs 1 through 10 in ATCM and ATCM+OTCM acquisitions for head and thorax. The location of ROIs is demonstrated in Fig. 1

\begin{tabular}{|c|c|c|c|c|}
\hline \multirow[b]{2}{*}{ ROI } & \multicolumn{4}{|c|}{ ATCM/ATCM+OTCM (\%Diff) } \\
\hline & Neonate & 1-year-old & 5-year-old & 10 -year-old \\
\hline \multicolumn{5}{|c|}{ Head } \\
\hline 1 & $4.21 / 4.47(6 \%)$ & $5.01 / 5.36(7 \%)$ & $5.04 / 4.85(-3 \%)$ & $5.58 / 5.66(1 \%)$ \\
\hline 2 & $4.86 / 4.35(-10 \%)$ & $5.51 / 6.09(10 \%)$ & $5.23 / 5.83(11 \%)$ & $5.86 / 5.91(1 \%)$ \\
\hline 3 & $4.96 / 4.64(-6 \%)$ & $6.24 / 6.86(10 \%)$ & $4.83 / 5.39(11 \%)$ & $7.42 / 7.68(3 \%)$ \\
\hline 4 & $4.21 / 5.51(30 \%)$ & $6.38 / 6.02(-5 \%)$ & $5.66 / 6.03(6 \%)$ & $6.40 / 6.72(5 \%)$ \\
\hline 5 & $6.07 / 7.34(21 \%)$ & $5.69 / 6.35(11 \%)$ & $5.24 / 5.58(6 \%)$ & $6.68 / 6.96(4 \%)$ \\
\hline 6 & $5.60 / 6.78(21 \%)$ & $5.07 / 5.88(16 \%)$ & $4.98 / 5.49(10 \%)$ & $6.37 / 6.99(9 \%)$ \\
\hline 7 & $6.12 / 5.74(-6 \%)$ & $4.74 / 5.42(14 \%)$ & $4.88 / 5.22(7 \%)$ & $5.97 / 6.58(10 \%)$ \\
\hline 8 & $5.89 / 6.19(5 \%)$ & $4.95 / 5.17(4 \%)$ & $4.47 / 5.00(11 \%)$ & $6.37 / 7.22(13 \%)$ \\
\hline 9 & $4.76 / 5.39(13 \%)$ & $4.87 / 5.71(17 \%)$ & $4.70 / 4.85(3 \%)$ & $5.87 / 6.84(16 \%)$ \\
\hline 10 & $4.48 / 6.45(43 \%)$ & $4.49 / 4.91(9 \%)$ & $4.20 / 5.64(34 \%)$ & $7.117 .25(2 \%)$ \\
\hline \multicolumn{5}{|c|}{ Thorax } \\
\hline 1 & $9.89 / 9.79(-1 \%)$ & $10.79 / 12.88(19 \%)$ & $10.99 / 12.50(13 \%)$ & $12.44 / 12.59(1 \%)$ \\
\hline 2 & $10.69 / 10.05(-6 \%)$ & $12.54 / 12.79(2 \%)$ & $8.66 / 12.39(43 \%)$ & $12.64 / 13.01(3 \%)$ \\
\hline 3 & $12.36 / 11.98(-3 \%)$ & $10.01 / 12.36(23 \%)$ & $13.29 / 15.34(15 \%)$ & $13.72 / 15.92(16 \%)$ \\
\hline 4 & $12.33 / 13.61(10 \%)$ & $10.88 / 14.40(32 \%)$ & $12.90 / 17.59(36 \%)$ & $14.56 / 14.62(0 \%)$ \\
\hline 5 & $12.43 / 11.97(-3 \%)$ & $12.72 / 14.85(16 \%)$ & $15.17 / 16.71(10 \%)$ & $14.36 / 16.54(15 \%)$ \\
\hline 6 & $12.36 / 13.69(10 \%)$ & $12.77 / 14.90(16 \%)$ & $14.66 / 16.72(14 \%)$ & $16.37 / 16.45(0 \%)$ \\
\hline 7 & $13.33 / 13.72(3 \%)$ & $12.36 / 13.88(12 \%)$ & $12.02 / 12.82(6 \%)$ & $14.00 / 14.87(6 \%)$ \\
\hline 8 & $11.49 / 13.72(19 \%)$ & $12.93 / 14.13(9 \%)$ & $11.29 / 13.13(16 \%)$ & $14.72 / 15.69(6 \%)$ \\
\hline 9 & $12.14 / 13.34(9 \%)$ & $12.70 / 14.10(11 \%)$ & $12.99 / 16.46(26 \%)$ & $15.80 / 16.55(5 \%)$ \\
\hline 10 & $11.26 / 12.22(8 \%)$ & $13.53 / 15.91(17 \%)$ & $14.24 / 16.49(15 \%)$ & $16.01 / 17.05(6 \%)$ \\
\hline
\end{tabular}

and $8 \%$ in spine for thorax [10]. This reduction was associated with a 6-20\% increase in image noise. Dixon et al, in a study using non-anthropomorphic phantoms, have shown that $\mathrm{CTDI}_{\mathrm{vol}}$ is reduced with an associated increase in image noise [11]. Herein, the dose to superficial and centrally located radiosensitive organs has been estimated using pediatric anthropomorphic phantoms. OTCM provokes a reduction in dose of all examined organs. This reduction is more significant for organs located within the OTCM-enabled image volume (Table 3). Large organs, such as lungs, in larger compared with smaller body sizes may fall partially outside the OTCM window. It would thus be reasonable to assume that dose for such organs might be less reduced. However, a trend of organ dose reduction with body size was not observed herein. This may be partly attributed to the different exposure parameters used for each anthropomorphic phantom and the limited number of examined body sizes.
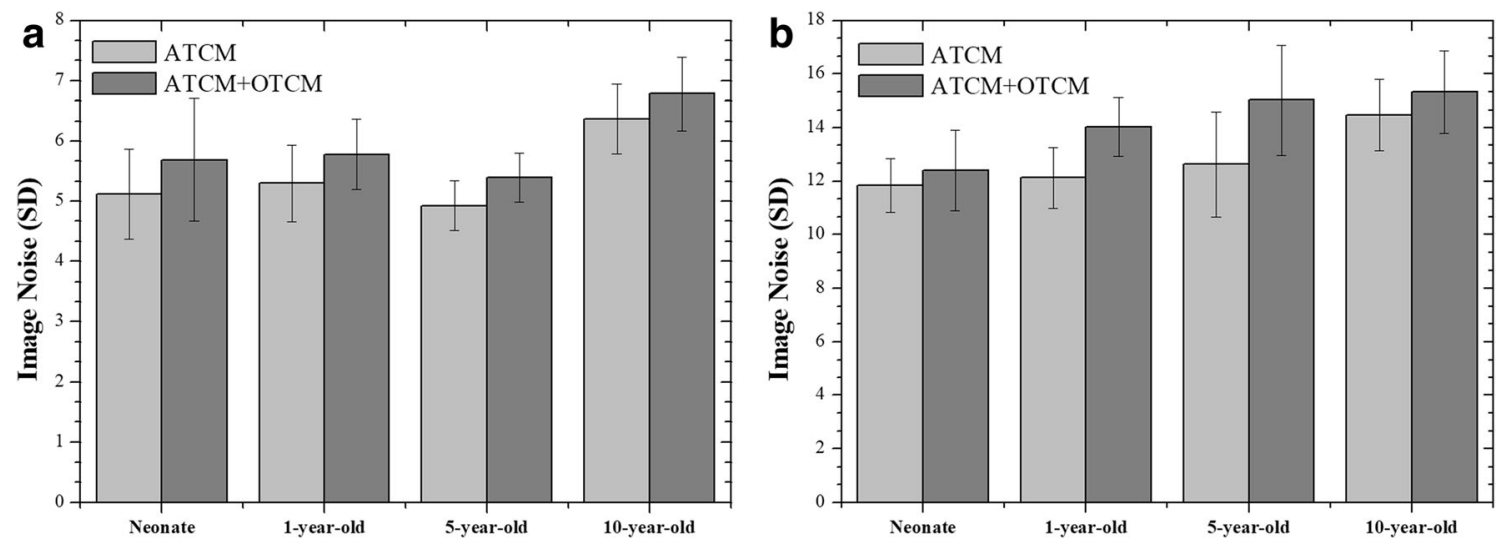

Fig. 5 Mean image noise values averaged over ROI 1 through ROI 10 at each anthropomorphic phantom for head (a) and thorax (b) acquisitions 
It should be noted that image quality should be thoroughly evaluated when dose reduction strategies are employed. Our results on noise measurements in slices within the OTCMenabled image volume showed that OTCM provokes a statistically significant but quantitatively small increase in noise (Table 5). Of note is that the \%Difference in noise varies strongly across the axial slice. There are also few ROIs where noise is reduced. Image noise increase might be higher in ROIs located in anterior locations of the slice where $\mathrm{mA}$ is reduced. However, such a trend was not observed herein. This is owing to the statistical behavior of noise, which may prevail over its dependence on $\mathrm{mA}$. Moreover, although the examined ROIs contain uniform soft tissue equivalent material, they are in close proximity with high attenuating bone structures which may affect the recorded noise through beam hardening. Gandhi et al, in an phantom study, have simulated a virtual ATCM+OTCM mode that employs the $\mathrm{mA}$ modulation scheme for the anterior $90^{\circ}$ or $180^{\circ}$ views, as prescribed by the OTCM used herein, but increases the $\mathrm{mA}$ for the remaining views [10]. As expected, this mode reduced dose for eye lens and breast to a lower, however, extent relative to real OTCM. It provided similar dose for lung and brain, and increased dose for spine. However, the increase in posterior $\mathrm{mA}$ did not fully recover image noise [10]. Whether or not the quantitatively small noise increase in images located within the OTCM-enabled volume is acceptable in the clinical practice is a matter of a further patient study. Of note is that image noise throughout the remaining image volume is expected to remain unaltered given that the applied $\mathrm{mA}$ values throughout this volume are governed by the ATCM modulation scheme, which is identical between the ATCM and ATCM+ OTCM. Patient dose might also be reduced in the ATCM mode by simply decreasing CTDI $_{\mathrm{vol}}$. For instance, to achieve the image noise increase of $15 \%$ found for thorax of the 1-year-old phantom, $\mathrm{CTDI}_{\mathrm{vol}}$ might be reduced by $\left[1-(1 / 1.15)^{2}\right] \times 100 \%=$ $24 \%$, which is considerably higher compared with $9 \%$ achieved with the ATCM+OTCM mode (Table 3). However, it should be emphasized that the $15 \%$ noise increase refers to every image within the series of the scanned volume.

This study has some limitations. First, clinical image quality was not evaluated. A further clinical study on a large number of pediatric patients at various ages and body sizes is required to investigate the effect of ATCM+OTCM on image quality. Second, image quality was assessed only on the image noise measure. A subjective evaluation of image quality from experienced pediatric radiologists is needed to assess the effectiveness of OTCM on generating images of diagnostic quality. Third, this study was limited to a single OTCM technique, which is available in the CT unit installed in our hospital.

In conclusion, our results showed that OTCM reduces radiation dose to all examined radiosensitive organs over the range of pediatric anthropomorphic phantoms. Eye lens in head and breast buds in thorax exhibit the highest dose reduction. However, OTCM increases image noise within the
OTCM-enabled imaged volume. Besides, we have characterized the tube radiation exposure when the OTCM acquisition mode is enabled in head and thorax of pediatric CT. Our results have shown that the low exposure window is substantially narrowed when short $t$ is used.

Acknowledgments This project has received funding from the Euratom research and training program 2014-2018 under grant agreement No 755523 (MEDIRAD). The funding source had no role in study design, in the collection, analysis and interpretation of data, nor in the writing or submission of the report.

Funding information This project has received funding from the Euratom research and training program 2014-2018 under grant agreement No 755523 (MEDIRAD).

\section{Compliance with ethical standards}

Guarantor The scientific guarantor of this publication is Antonios E Papadakis.

Conflict of interest The authors of this manuscript declare no relationships with any companies whose products or services may be related to the subject matter of the article.

Statistics and biometry No complex statistical methods were necessary for this paper.

Informed consent Written informed consent was not required for this study because phantoms were exclusively used.

Ethical approval Institutional Review Board approval was not required because this study was performed on phantoms.

\author{
Methodology \\ - prospective \\ - experimental \\ - performed at one institution
}

Open Access This article is licensed under a Creative Commons Attribution 4.0 International License, which permits use, sharing, adaptation, distribution and reproduction in any medium or format, as long as you give appropriate credit to the original author(s) and the source, provide a link to the Creative Commons licence, and indicate if changes were made. The images or other third party material in this article are included in the article's Creative Commons licence, unless indicated otherwise in a credit line to the material. If material is not included in the article's Creative Commons licence and your intended use is not permitted by statutory regulation or exceeds the permitted use, you will need to obtain permission directly from the copyright holder. To view a copy of this licence, visit http://creativecommons.org/licenses/by/4.0/.

\section{References}

1. International Commission on Radiological Protection (2011) Statement on tissue reactions ICRP. Ref. 4825-3093-1464

2. International Commission on Radiological Protection (2007) The 2007 recommendations of the International Commission on 
Radiological Protection. ICRP Publication 103. Ann ICRP 37(24):1-332

3. Chodick G, Bekiroglu N, Hauptmann M et al (2008) Risk of cataract after exposure to low doses of ionizing radiation: a 20-year prospective cohort study among US radiologic technologists. Am J Epidemiol 168(6):620-631

4. Worgul BV, Kundiyev YI, Sergiyenko NM et al (2007) Cataracts among Chernobyl clean-upworkers: implications regarding permissible eye exposures. Radiat Res 167(2):233-243

5. World Health Organization (2020) World cancer report: cancer research for cancer prevention. World Health Organization, Lyon. Available via http://publications.iarc.fr/Non-Series-Publications/ World-Cancer-Reports/World Cancer-Report-Cancer-ResearchFor-Cancer-Prevention-2020. Accessed 10 Feb 2020

6. Franck C, Smeets P, Lapeire L, Achten E, Bacher K (2018) Estimating the patient-specific dose to the thyroid and breasts and overall risk in chest CT when using organ-based tube current modulation. Radiology 288(1):164-169

7. Duan X, Wang J, Christner JA, Leng S, Yu L, McCollough CH (2011) Dose reduction to anterior surfaces with organ-based tubecurrent modulation: evaluation of performance in a phantom study. AJR Am J Roentgenol 197(3):689-695

8. Fu W, Tian X, Sturgeon GM et al (2017) CT breast dose reduction with the use of breast positioning and organ-based tube current modulation. Med Phys 44(2):665-678

9. Wang J, Duan X, Christner JA, Leng S, Yu L, McCollough CH (2011) Radiation dose reduction to the breast in thoracic CT: comparison of bismuth shielding, organ-based tube current modulation, and use of a globally decreased tube current. Med Phys 38(11): 6084-6092

10. Gandhi D, Crotty DJ, Stevens GM, Schmidt TG (2015) Technical note: phantom study to evaluate the dose and image quality effects of a computed tomography organ-based tube current modulation technique. Med Phys 42(11):6572-6578

11. Dixon MT, Loader RJ, Stevens GC, Rowles NP (2016) An evaluation of organ dose modulation on a GE optima CT660-computed tomography scanner. J Appl Clin Med Phys 17(3):380-391

12. Euler A, Szucs-Farkas Z, Falkowski AL et al (2016) Organ-based tube current modulation in a clinical context: dose reduction may be largely overestimated in breast tissue. Eur Radiol 26(8):2656-2662

13. Taylor S, Litmanovich DE, Shahrzad M, Bankier AA, Gevenois PA, Tack D (2015) Organ-based tube current modulation: are women's breasts positioned in the reduced-dose zone? Radiology 274(1):260-266

14. Boos J, Kröpil P, Klee D et al (2014) Evaluation of the impact of organ-specific dose reduction on image quality in pediatric chest computed tomography. Pediatr Radiol 44:1065-1069

15. Yamauchi-Kawaura C, Yamauchi M, Imai K, Ikeda M, Aoyama T (2013) Image quality and age-specific dose estimation in head and chest CT examinations with organ-based tube-current modulation. Radiat Prot Dosimetry 157(2):193-205

16. Papadakis AE, Perisinakis K, Damilakis J (2014) Automatic exposure control in CT: the effect of patient size, anatomical region and prescribed modulation strength on tube current and image quality. Eur Radiol 24(10):2520-2531

17. Papadakis AE, Perisinakis K, Raissaki, Damilakis J (2013) Effect of $\mathrm{X}$-ray tube parameters and iodine concentration on image quality and radiation dose in cerebral pediatric and adult CT angiography: a phantom study. Invest Radiol 48(4):192-199

18. User Manual, Revolution Discovery CT (2014) 5507105-1EN Rev1. 1056-1057

19. Papadakis AE, Damilakis J (2019) Automatic tube current modulation and tube voltage selection in pediatric computed tomography: a phantom study on radiation dose and image quality. Invest Radiol 54(5):265-272

20. Papadakis AE, Perisinakis K, Damilakis J (2016) Development of a method to estimate organ doses for pediatric CT examinations. Med Phys 43(5):2108-2117

21. Perisinakis K, Seimenis I, Tzedakis A, Papadakis AE, Damilakis J (2012) Triple-rule-out computed tomography angiography with 256-slice computed tomography scanners: patient-specific assessment of radiation burden and associated cancer risk. Invest Radiol 47:109-115

22. Deak P, van Straten M, Shrimpton PC, Kalender WA (2008) Validation of a Monte Carlo tool for patient-specific dose simulations in multi-slice computed tomography. Eur Radiol 18:759-772

23. Perisinakis K, Seimenis I, Tzedakis A, Papadakis AE, Damilakis J (2010) Individualized assessment of radiation dose in patients undergoing coronary computed tomographic angiography with 256slice scanning. Circulation 122(23):2394-2402

24. Inkoom S, Raissaki M, Perisinakis K, Maris TG, Damilakis J (2015) Location of radiosensitive organs inside pediatric anthropomorphic phantoms: data required for dosimetry. Phys Med 31(8): 882-888

25. Lungren MP, Yoshizumi TT, Brady SM et al (2012) Radiation dose estimations to the thorax using organ-based dose modulation. AJR Am J Roentgenol 199(1):W65-W73

26. Ketelsen D, Buchgeister M, Fenchel M et al (2012) Automated computed tomography dose-saving algorithm to protect radiosensitive tissues: estimation of radiation exposure and image quality considerations. Invest Radiol 47(2):148-152

27. Wang J, Duan X, Christner JA, Leng S, Grant KL, McCollough CH (2012) Bismuth shielding, organ-based tube current modulation, and global reduction of tube current for dose reduction to the eye at head CT. Radiology 262:191-198

Publisher's note Springer Nature remains neutral with regard to jurisdictional claims in published maps and institutional affiliations. 